\title{
Evidenzen, Indizien und Beweise
}

\author{
Der Alltag im himelrîche zwischen Philologie und \\ Kulturwissenschaft
}

Das Problem an den Turns in den Geisteswissenschaften ist, dass sie keine wirklichen Turns sind. Sie reagieren nicht auf neue Fakten, sie sind keine Kopernikanischen Wenden. Sie eröffnen neue Perspektiven, ohne die alten wirklich außer Kraft zu setzen. Sie sind >Wendungen`, keine >Wenden`. Die Reaktionen auf die Turns sind entsprechend. Die Vertreter der Turns sind euphorisch und sie müssen es sein, denn eigentlich macht erst diese Euphorie den Turn aus. Man tut so, als habe sich die Wissenschaft und das Wissen um die Welt substantiell verändert, und radikalisiert damit die Geltung einer neuen Perspektive, auch wenn sie die alten nicht ablöst. Das Problem dabei ist, dass sich Euphorie nicht auf Dauer stellen lässt, bzw. nur mit größten Glaubwürdigkeitsverlusten; wer es trotzdem versucht, wird zwangsläufig unglaubwürdig und man kennt ja die Veteranen in die Jahre gekommener Innovationen. Aber auch die Skeptiker gegenüber den Turns können nicht uneingeschränkt agieren: So recht eigentlich

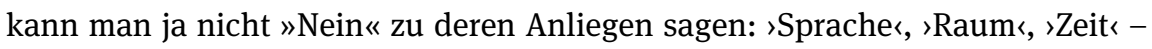
aber auch Dimensionen wie `Geschlecht`, `Materialität`, etc. kann man ja nicht für bedeutungslos erklären. Deshalb kommt statt einem »Nein « oft ein »Ja, aber«. Die Gegner der New Philology etwa reagierten mit dem Hinweis, dass sie die Materialität ohnehin schon immer für zentral gehalten haben, »Ja«, aber neu sei die Sache wirklich nicht, jetzt nur irgendwie amerikanisch. ${ }^{1}>$ Kulturwissenschaftく, »Ja«, das sei die Germanistik natürlich schon seit den Grimms, doch irgendwie anders.

Weil die Turns also die Welt nicht verändern, aber auch nicht an der Welt vorbeigehen, werden sie weder radikal verifiziert noch falsifiziert, sondern ebben ab. Sie hinterlassen dabei aber doch die Aspekte, die sie ins Zentrum

1 Vgl. etwa Freimut Löser: Postmodernes Mittelalter? `New Philology und Überlieferungsgeschichte. In: Jochen Conzelmann u. a. (Hg.): Kulturen des Manuskriptzeitalters. Ergebnisse der Amerikanisch-Deutschen Arbeitstagung an der Georg-August-Universität Göttingen vom 17. bis 20. Oktober 2002. Göttingen 2004, S. 215-236.

Stephan Müller, Wien

Ә Open Access. (C) 2022 Stephan Müller, publiziert von De Gruyter. (cc) BY Dieses Werk ist lizenziert unter einer Creative Commons Namensnennung 4.0 International Lizenz. https://doi.org/10.1515/9783110667004-003 
stellten. Die Fragen nach diesen Aspekten liegen dann in der Werkzeugkiste der Textarbeiterinnen und Textarbeiter und können mit Gewinn zur Anwendung

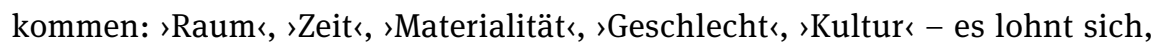
danach zu fragen. Das Problem ist nur, dass damit recht unterschiedliche Instrumente im Werkzeugkasten liegen: Skalpell liegt neben Kettensäge - mit beiden kann man sinnvoll Schnitte setzen, aber eben nicht in allen Situationen gleich sinnvoll.

Ich lasse dieses Bild im Raum stehen und wende mich in diesem Sinn meinem Text zu. Ich will dabei versuchen, auf verschiedenen Ebenen und mit verschiedenen Methoden auf diesen Text zuzugreifen und $\mathrm{zu}$ fragen, wie die Ergebnisse dieser Zugriffe miteinander in Bezug zu setzen sein könnten. Ansetzen werde ich dabei mit der Beschreibung von Auffälligkeiten, die den Text prägen und ich will diese Auffälligkeiten versuchsweise in einem ersten Schritt als Evidenzen bezeichnen, um diese dann sukzessive zusammenzusehen. Unter dem Begriff Text wird selbstverständlich seine Materialität bedeutungshaft mit verstanden, ohne dass ich meine, das begründen zu müssen - was ein Beispiel dafür sein mag, wie eine vergangene rege Debatte (um Überlieferungsgeschichte und New Philology) als Teil wissenschaftlicher Praxis inventarisiert wurde. Die Leitfrage wird sein, ob und wie diese Evidenzen in verschiedenen Zusammenhängen zum Argument werden können. Es geht also dabei zunächst um nicht mehr, als um das Benennen von Auffälligkeiten in der Befundlage und das ohne gleich schon in den Prozess einer Deutung überzugehen (um das alte Paradigma von »Befund und Deutung « hier aufzurufen und im selben Atemzug zu dispensieren). Erst resümierend will ich dann fragen, was die Voraussetzungen dafür sind, dass Evidenzen zu Argumenten werden und ob diese Voraussetzungen etwas darüber aussagen, wie sich philologische Befunde zu kulturwissenschaftlichen Analysen verhalten.

Bei meinem Gegenstand handelt es sich um einen Text, der die Themen `Himmlisches Jerusalem`, >Jüngstes Gericht` und in diesem Kontext eine Allegorese des Regenbogens behandelt. Er umfasst in der gängigen Edition ${ }^{2} 378$ Langverse, firmiert in der Forschung unter dem Titel Daz himelrîche und entstand zwischen 1140 und 1180, wobei einiges für eine späte Datierung in diesem Zeitraum spricht. ${ }^{3}$

2 Vgl. Vom Himmelreich. In: Die religiösen Dichtungen des 11. und 12. Jahrhunderts. Nach ihren Formen besprochen und hg. von Friedrich Maurer, Band 1. Tübingen 1964, S. 374-395. 3 Vgl. Wiebke Freytag: ১Daz himelrîcheく. In: ${ }^{2}$ VL 4 (1983), Sp. 18-21. 
Literaturgeschichtlich bewertete man den Text mal als stümperhaft, ${ }^{4}$ mal ist der Autor "wirklicher Dichter " ${ }^{5}$ und ordnet ihn im Sinne einer etablierten, aber seltsamen Asynchronie ein: Die Texte der zweiten Hälfte des 12. Jahrhunderts sind frühe Texte, wenn sie sweltlich ‘ sind, sie sind späte Texte, wenn sie 'geistlich s sind. Veldekes Eneit ist >frühhöfisch`, die weltlichen Texte streben der Zeit um $1200 \mathrm{zu}$. Unser Text ist dagegen etwa nach Helmut de Boor »spätcluniazensisch ${ }^{6}{ }^{6}$ also Ausläufer einer Tradition, die für de Boor im 11. Jahrhundert begann. In meiner Versuchsanordnung will ich den Text aber aus solchen literarhistorischen Kontinuen herausnehmen und einfach in meinen Werkzeugkasten greifen, um ihn für sich selbst genommen zu beschreiben.

\section{Die Evidenz des Materials}

Damit zu meiner ersten Evidenz, der Evidenz des Materials: Daz himelrîche steht im Clm 9513 der Bayerischen Staatsbibliothek, einem Codex aus Oberaltaich, am Blattrand von folio 1 recto bis 7 recto neben dem elften Buch von Gregors des Großen Moralia in Iob. Die Eintragung ist geplant, wie eine Bildlinierung auf den Rändern der Handschrift zeigt. Sie ist aber auch gestört. Am Ende werden Textteile mit Verweiszeichen nachgetragen. ${ }^{7}$

Nun kann man die Hand des deutschen Textes eindeutig identifizieren, und zwar als jene, die auch die berühmte Interlinearversion des Windberger Psalters

4 Vgl. Rudolf Hävemeier: Daz himilriche. Ein bairisches Gedicht aus dem 12. Jahrhundert. Bückeburg 1891, S. 18-20 fasst die Urteile von Scherer und Steinmeyer zusammen. Scherer ist ganz ungnädig. Steinmeyer entschuldigt immerhin die Qualität mit der offensichtlichen Jugend des Dichters, was Hävemeier nicht für angemessen hält, weil er im Autor einen »gereisten Mann« (S. 19) vermutet.

5 Helmut de Boor: Die deutsche Literatur von Karl dem Großen bis zum Beginn der höfischen Dichtung 770-1170. 6. Aufl. München 1964 (Geschichte der deutschen Literatur von den Anfängen bis zur Gegenwart 1), S. 192.

6 Bei de Boor: Die deutsche Literatur (s. Anm. 5) steht Daz himelrîche im Kapitel »Cluniazensische Spätzeit« (S. 172-199).

7 Zur Überlieferungssituation vgl. Stephan Müller: Willkomm und Abschied. Zum problematischen Verhältnis von `Entstehung ‘ und `Überlieferung` der deutschen Literatur des Mittelalters am Beispiel von »Ezzolied«, »himelrîche« und »Vorauer Handschrift«. In: Jens Haustein, Helmut Tervooren (Hg.): Regionale Literaturgeschichtsschreibung. ZfdPh Sonderheft zum Band 122 (2003), S. 230-245 und Christine Stridde: Verbalpräsenz und göttlicher Sprechakt. Zur Pragmatik spiritueller Kommunikation >zwischen`St. Trudperter Hohelied und Mechthilds von Magdeburg Das fließende Licht der Gottheit. Stuttgart 2009, S. 90-92. 
im Cgm 17 geschrieben hat. ${ }^{8}$ Diesen eindeutigen Befund verdanken wir Karin Schneider, die für die Paläographie vor allem auch deutschsprachiger Schriften des Hochmittelalters die aktuellen Standards gesetzt hat. ${ }^{9}$ Das führt aber zu der Frage, was eine Windberger Hand in einem Oberaltaicher Codex zu suchen hat? Es gibt mehrere Antwortmöglichkeiten: Entweder schrieb ein Windberger Prämonstratenser im Benediktinerkloster Oberaltaich, oder der Codex war nach Windberg ausgeliehen, wo man den Text der Moralia abschrieb und dann das deutsche himelrîche - vielleicht als Geschenk für die Oberaltaicher - eintrug. Als Produkt dieser Abschrift der Moralia kämen nur der Clm 22202 und 22203 in Frage, die in Windberg den Text der Moralia überliefern. Seitdem die Untersuchungen von Brigitte Pfeil zeigen konnten, ${ }^{10}$ dass nur der Oberaltaicher Clm 9513 Vorlage für die Windberger Moralia war, nicht dagegen der erste Band der Oberaltaicher Moralia, der Clm 9512, ist besser erklärbar, warum das himelrîche nicht am Anfang der Moralia eingetragen wurde, sondern neben dem elften Buch und man weiß nun auch, dass man in Windberg zwei Vorlagen verwendete, woraus man folgern kann, dass der Oberaltaicher Codex erst später ausgeliehen wurde und man wohl länger an der Abschrift arbeitete und vielleicht das himelrîche als kleines Dankeschön »wahrscheinlich auf Bitten der Oberaltaicher ${ }^{11}$ in die Oberaltaicher Handschrift eintrug.

Wie genau der Austausch auch immer vor sich ging, sicher ist Folgendes: Der Codex und damit auch unser himelrîche wird im Kontext der Benediktiner und Prämonstratenser gebraucht. Und: Der Text hat eine kalkulierte Überlieferungsqualität. Es ist keine spontane Niederschrift vor Ort und zwar eine Niederschrift eines Schreibers, der nicht nur diesen deutschen Text schrieb, sondern auch den Windberger Psalter. Diese eindeutige paläographische Zuschreibung verlangte also nach Erklärungen, bei denen eindeutige Befunde der Textkritik (nämlich der Vorlagenverhältnisse der Windberger Moralia) und der Kunstgeschichte herangezogen wurden, um dann daraus einen möglichen Schluss zu

\footnotetext{
8 Ich zitiere den Text nach Klaus Kirchert: Der Windberger Psalter, Bd. II: Textausgabe. Zürich 1979.

9 Vgl. Karin Schneider: Gotische Schriften in deutscher Sprache: Vom späten 12. Jahrhundert bis um 1300; Bd. 1: Textband; Bd. 2: Tafelband. Wiesbaden 1987, hier Bd. 1, S. 34. Schon Johann Andreas Schmeller hat in seiner Erstausgabe von 1851 (in ZfdA, Bd. 8, S. 145-155) eine Schreiberidentität als »höchst wahrscheinlich « (S. 145) angesehen.

10 Vgl. Brigitte Pfeil: Die >Vision des Tnugdalus` Albers von Windberg. Literatur- und Frömmigkeitsgeschichte im ausgehenden 12. Jahrhundert. Mit einer Edition der lateinischen $>$ Visio Tnugdali ‘ aus Clm 22254. Frankfurt a.M. u. a. 1999, S. 55 f. Der Clm 9513 ist nach Ausweis der ikonographischen Untersuchungen von Elisabeth Klemm auch teilweise Vorlage für den Clm 22203. Vgl. Stridde (Anm. 7), S. 74, Anm. 286.
}

11 So Klaus Kirchert: Der Windberger Psalter, Bd. I: Untersuchungen. Zürich 1979, S. 63. 
ziehen. Wie die Verbindung zwischen Windberg und Oberaltaich genau aussah und in welchen Kontexten die eindeutige Kenntnis dieser Verbindung zum Argument werden könnte, ist damit nicht gesagt. Aber man kann sich entsprechende ordensgeschichtliche Untersuchungen gut vorstellen, in denen dieser Befund zum Argument werden könnte. Für den vorliegenden Versuch reicht es, auf die Evidenz der Schreiberindentität zu setzen. Sie belegt, dass ein und dieselbe Person zwei Mal deutsche Texte schrieb und dass einer dieser Texte kein spontanes Autograph ist, sondern Produkt einer geplanten Überlieferung und Weitergabe.

\section{Die Evidenz der Sprache}

Damit zu einer daraus folgenden Evidenz - zur Evidenz der Sprache: Der Text des himelrîche beginnt mit dem Eingang von Psalm 47: Mihil bis du herro got unde loebelich harte $(1,1)$ (Groß bist Du, Herr, und sehr lobenswert) übersetzt: Magnus dominus et laudabilis nimis. Im Windberger Psalter (S. 88) schreibt dieselbe Hand das so: Michil herro unde loblih harte - also fast wortgleich. Aufgerufen wird am Anfang des himelrîche also Psalm 47, doch die Übersetzung weicht einer Paraphrase: michil ist din chraft uf dere himilisken warte $(1,2)$ (Groß ist deine Kraft auf der himmlischen Warte). Die shimmlische Warte`, das Reich, das über allen anderen Reichen steht, ist im folgenden Text dann das >Himmlische Jerusalem . Das Psalmenzitat mündet also sofort in das zentrale Motiv des himelrîche, ins >Himmlische Jerusalem`, von dem allerdings im Psalm 47 nicht die Rede ist! Und auch die exegetische Tradition legt den Psalm 47 nicht Richtung >Himmlisches Jerusalem aus. $^{12}$

Die Verbindung stellt indes eine Oratio zum Psalm her. ${ }^{13}$ Solche Orationes sind im Windberger Psalter jedem Psalm beigegeben und werden ebenfalls interlinear ins Deutsche übersetzt. Für Psalm 47 beginnt sie wie folgt: Amabilis atque laudabilis deus, qui in caelesti hierusalem princeps magnificus inveneris. (S. 90) Für uns ist nun wichtig, dass dieser Satz der Oratio im Windberger Psalter wie folgt übersetzt wird: Minnichlih iouh lobelih got du der in der himilisken frideburge furste ein michillih funden wirdis. (S. 90) (Liebenswert und auch lobenswert bist Du Gott, der du in der himmlischen Friedensstadt - also im Himmlischen Jerusalem - als ein großer Herrscher angetroffen wirst).

12 Als Auslegungstraditionen kämen die Kommentare des Cassiodor und Augustin in Frage, die beide das Himmlische Jerusalem nicht erwähnen.

$13 \mathrm{Zu}$ den Orationes vgl. Kirchert: Untersuchungen (s. Anm. 11), S. 79-81. 
Das >Himmlische Jerusalem heißt hier, wie es oft heißt: himilisike frideburge. In den Eingangsversen des himelrîche ist dagegen von der himilisken warte die Rede. Das sticht besonders ins Auge, wenn man sieht, dass warte im Kontext des 47. Psalms den expliziten Bezug zu einem anderen Ort herstellt, der im Windberger Psalter immer mit »Warte« wiedergegeben wird: Mit dem Berg Zion, der im zweiten Vers des Psalm 47 als die Stadt des größten Königs gepriesen wird. Der Mons Sion wird mit berg warte übersetzt und in der Oratio dann mit dem Jerusalem Caelestis gleichgesetzt. Das himelrîche kombiniert also eine Bezeichnung, die den Namen des Bergs Zion aufgreift (warte) und fügt die Deutung der Oratio hinzu: So entsteht aus des berges warte und der himilisken frideburge die himiliske warte - also eine aus der Psalmenexegese der Oratio abgeleitete Form für das »Himmlische Jerusalem«.

Eine solche Engführung von Wortschatzarbeit und Exegese ist die Regel auch im Windberger Psalter. Oft wird sie durch das einfache Nebeneinander mehrerer Lexeme geleistet, die im Windberger Psalter bis zum Exzess praktiziert wird. Ein beliebiges Beispiel: In Psalm 17,16 wird fontes aquarum zweimal übersetzt und von diesen Übersetzungen dann eine durchgestrichen: Einmal als ursprinch der wazzere, einmal als brunnen der wazzere. (S. 31) Es handelt sich im Text um eine große Theophanie, mit der David schildert, wie Gott ihm gegen seine Feinde zu Hilfe kommt. Man kann die fontes wörtlich natürlich als `Quelle lesen - wie in der Übersetzung brunnen, was die Drastik des Bildes aber unterschlägt. Die Übersetzung mit >Ursprung، ist abstrakter und korrespondiert mit der Auslegung des Psalms durch Cassiodor, der die fontes als Begründung für die Heiligkeit des ewigen Lebens liest, weshalb Notker der Deutsche, der hier Cassiodor benutzt, das fontes - wie in Windberg - mit urspringa übersetzt. ${ }^{14}$ In Windberg ist diese Übersetzung zwar durchgestrichen, aber eben nicht getilgt. Sie bleibt gut lesbar, sodass eine Abwägung von Deutungs- und Übersetzungsalternativen (und die Entscheidung, die auf diesen Prozess folgt) nachvollziehbar bleibt. Wortsinn und exegetische Deutung sind kopräsent. Solche Synonymnester können bis zu fünf Lexeme vereinen und auch im himelrîche stehen in exzessiver Häufigkeit Synonyme nebeneinander. Schon im vierten Vers heißt es etwa: dinen gewalt mach niemen enphliehen noch entwichen. (1,4) (Deiner Macht kann niemand entfliehen noch entweichen). In Vers sechs folgt dann als Reimwort noch das synonyme entrinnen. $(1,6)$ Auch im Windberger Psalter wird ein Naturbild in Psalm 74,7 erklärt mit: maget ir entrinnen. oder daz gerihte enphliehen (S. 138) (könnt ihr dem Gericht nicht entrinnen noch entweichen).

14 Vgl. Notker der Deutsche: Der Psalter. Psalm 1-50. Hg. von Petrus W. Tax. Tübingen 1979 (Die Werke Notkers des Deutschen. Neue Ausgabe 8, ATB 84), S. 50, Zeile 12. 
Die Synonymnester sind dabei mehr als nur eine Marotte, sie prägen einen repetitiven Stil, der in der »Interpretationstechnik des Psalters « seine »Entsprechung " hat ${ }^{15}$ und sind also auch ein Medium von Sprach- und Weltreflexion, was bewirkt, dass im himelrîche, wie im Windberger Psalter zahllose Frühstund Einzelbelege mittelhochdeutscher Lexeme zu finden sind. Auch das nur kurz: Über Butyrum (im 'Canticum Moisi’ [Deut. 32,14], Cgm 17, fol. 213r, Z. 5-7;) stehen die Übersetzungen Die cigeren. buttiren. anchsmere. Butyrum wird mit dem ab dem 11. Jahrhundert belegten Lehnwort buttir, mit dem Erstbeleg des Wortes ciger und mit einem sehr frühen Beleg des erst im 12. Jahrhundert überlieferten anchsmere übersetzt. Wie benennt man Dinge des Alltags? Die Frage stellt man, aber entscheidet sich nicht, sondern stellt Alternativen vor. Die so entstehenden Synonymnester integrieren eine (hier lexikalische) Reflexion des Alltags in die Welt monastischer Spiritualität. Dies alles, um daran zu erinnern, aus der Hand desselben Schreibers und als Text, der nicht nur einmal aufgeschrieben wird, sondern den man weitergibt.

\section{Die Evidenz des Alltags}

Damit zur nächsten Evidenz, der Evidenz des Alltags: Der Zustand des >Himmlischen Jerusalem « wird im himelrîche durch die Abwesenheit von Alltagsnotwendigkeiten beschrieben. Als »Schilderung durch Negation « hat man das wiederholt bezeichnet. ${ }^{16}$ Das ist ein oft belegtes Muster und keine Besonderheit. Im Falle des himelrîche aber ist dieses Muster ein Einfallstor für eine sprachliche Abbildung des Alltags, über den sonst nicht geschrieben würde und dies - wie in der Evidenz der Sprache gezeigt - im Modus sprachlicher Reflexion. Ein Beispiel (9,1f.):

\footnotetext{
Ire gewaete, die da sint, ist das ewige lieht, vone diu nebedurfen si dere badegwante alanch nieht.

(Ihre Gewänder sind dort das ewige Licht, deshalb brauchen sie keine Badegewänder.)
}

15 Gisela Vollmann-Profe: Wiederbeginn volkssprachlicher Schriftlichkeit im hohen Mittelalter (1050/60-1160/70). Königstein im Taunus 1986 (Geschichte der deutschen Literatur von den Anfängen bis zum Beginn der Neuzeit. Band I: Von den Anfängen bis zum hohen Mittelalter. Teil 2), S. 146. Vgl. auch Freytag (Anm. 3), Sp. $19 \mathrm{f}$.

16 So etwa Hävemeier: Daz himilriche (s. Anm. 4), S. 19. 
Weil die Seligen im >Himmlischen Jerusalem` als Kleidung das ewige Licht tragen, brauchen sie keine Badehosen. Auch hier neigt der Text zum Exzess und auch wieder zur Bildung der Synonymnester (9,12ff.):

durh ezzen nebedarf man daz brot bachen noch baen,
durh zuomuose fleisc und(e) viske sieden noch sulzen,
durh trinchen haberen noch gersten ze bierè mulzen.
sí negerent durh den durst iemer metes noch wines
oder ze wollibe morates noch trinchenes deheines.
(Man muss das Brot zum Essen nicht backen oder rösten,
Fleisch und Fische als Zuspeise weder kochen noch sülzen,
zum Trinken den Hafer noch die Gerste zu Bier malzen.
Sie begehren aus Durst weder Met noch Wein
noch für ihr Wohlbefinden Maulbeerwein oder irgendein Getränk.)

und 9,22ff:

ane strælære unde bursten wirdit in daz har geslihtit. si ruowent da ane vederbete, bolstære unde chusse, nehein wert hat der choze da also vile so diu zusse. undurft ist in lichlachen jouch dere bedelachen, si sint is alles vervángèn mit geistlichen sachen.

(Ohne Kämme und Bürsten wird ihnen das Haar geglättet.

Sie ruhen dort ohne Federbetten, Polster und Kissen, der Lodenkotzen hat dort so wenig Funktion wie der Lodenumhang. Unnötig sind für sie Leinentücher und Bettlaken, sie sind ganz mit geistlichen Dingen umfangen)

Hier hagelt es förmlich Frühstbelege und Sonderwortschatz: baen (»in Scheiben geschnittenes Brot leicht rösten«) - sulzen (»einsülzen«) - mulzen (»malzen«) der Morat, der "Maulbeerwein « - zusse und chozze, zwei Wörter für den »Lodenkotzen«, strælær der »Kamm « oder »Kammmacher« - »Bettlaken« - »Federbett« - »Polster« - »Kissen«. Die Beispiele ließen sich vermehren. Solche Thematisierungen des Alltags gehen auch über die Ebene des Einzelwortes hinaus. Als Beispiel dafür ein Scholion - also ein erläuternder Randeintrag zu Jubilare im Windberger Psalter (zu Psalm 94,2 [S. 177]). Dort wird der Jubel wie gewöhnlich als Form der Sprache ohne Wortsinn erklärt. Mit den Worten des Scholions als ein sang ane wort. Aber das wird in Windberg nun zusätzlich rückgebunden an die Alltagserfahrung der Rezipienten: so ir ofte uernomen habet. uon den geburen. iouh uone den chindelinen die dennoh dere worte. gebiliden neweder nemagen. nohne chunnen. (S. 177) (Das habt ihr oft bei den Bauern und Kindern gehört, die keinen rechten Wortsinn zu bilden vermögen und können). Die Alltagserfahrung ist also Gegenstand der exegetischen Textpraxis, soweit die Evi- 
denz, aber nicht nur der Alltag ist Gegenstand der Exegese. Der Windberger Text operiert so mit exegetischen Quellen, wie das in der Zeit üblich ist.

\section{Die Evidenz der Quellen}

Damit zur Evidenz der Quellen. Wir sahen bereits im Eingangsvers, dass himelrîche und Oratio zum Psalter interagieren. Darüber hinaus hat die Forschung eine Reihe von Quellen namhaft gemacht. ${ }^{17}$ Deutlich und sehr üblich ist dabei die Verwendung des Honorius Augustodunensis. Man bezeichnet Isidors Etymologien oft als `Brockhaus des Mittelalters`. In der Logik eines solchen Vergleichs könnte man Honorius >Wikipedia des Mittelalters` nennen. Seine Schriften sind meist nicht originell, sondern oft eine wilde Zusammenschau zahlreicher Quellen. Werke wie De imago mundi oder, noch breiter, seine Summa totius de omnimoda historia stellen diesen Anspruch schon im Titel aus. In Windberg wurden seine Werke im 12. Jahrhundert geschrieben und auch im himelrîche aktiv benutzt. Besonders prägend war dabei sein Elucidarium, ein fingierter Dialog zwischen Lehrer und Schüler, in dem nach dem Wissen um Gott und die Welt gefragt wird. Das himelrîche entnimmt ihm etwa die Differenzierung von drei Reichen - von driu riche $(2,5)$ - und auch bei der Allegorese des Regenbogens, die ich jetzt besprechen will, hat der Text Pate gestanden.

Es kommt mir darauf an zu zeigen, dass im Text zwar Quellen aufgerufen, diese aber dann auch in Relation zur eigenen Erfahrung gesetzt werden. Textpraxis und Lebenspraxis interagieren. Die Regenbogenallegorese beginnt dabei betont defensiv: Das >Ich gemäß zu deuten und thematisiert die Gefahr, daz ich verliese die arbeite minere anedæhte $(5,9)$ (dass ich die Mühen meiner Andacht verliere). Also den Gewinn seines kontemplativen Lebens - der anedæhte - zu verspielen. Diese Gefahr ist wichtig und schwingt im Text immer mit. In dieser Situation zieht sich das >Ich auf Quellen zurück: Auf das Wissen der buochmeister $(4,26)$, das man als gegeben hinnimmt, aber auch auf die Aussagen des Lehrers:

doch sage ich, daz mich $g(e)$ leret hat min magezoge, von gehilwe unde heitere wirt der regenboge

(Doch ich sage, was mich mein Lehrer gelehrt hat:

Aus Wolken und heiterem Himmel entsteht der Regenbogen).

17 So detailliert schon in der Arbeit von Hävemeier: Daz himilriche (s. Anm. 4), S. 31 f. 
Das zweifelnde >Ich` setzt sich über die Gefahren der Unwahrheit hinweg (doch) und privilegiert dabei den eigenen Lehrer als Quelle. Die Aussage des Lehrers steht in einer breiten Tradition: Wolken (gehilwe) und Sonne (heitere) erzeugen den Regenbogen. Zwei Elemente also - sunnen wirmen und wolchen fiuhte (die Wärme der Sonne und die Feuchtigkeit der Wolken), fiure und wazzer (Feuer und Wasser) (5,12-14) - lassen den Regenbogen entstehen. Diese beiden Phänomene lässt das >Ich des Textes dann mit einem dritten korrespondieren:

daz dritte dar unter ist des himiles varwe gluche, ich wæne, in diu erde an ietwederem orte zuo sich luche.

$(5,15 f$.

(als Drittes kommt die glänzende Farbe des Himmels dazu, ich glaube die Erde zieht ihn an jedem Ort an sich.)

Hier meldet sich die vorher so bescheidene >Ich--Instanz selbst zu Wort und bezeichnet die leuchtende Himmelsfarbe als ein Drittes, das aus den beiden ersten Phänomenen hervorgehe. Dem fügt das `Ichı eine Vermutung hinzu (ich wæne, 5,16), die ich mit Lexer so verstehe: `Ich vermute, dass die Erde ihn (den Regenbogen) an jedem Ort an sich zieht،. Diese Spekulationen zum Himmelslicht und den Orten auf der Erde bindet das Phänomen des Regenbogens an die vier Elemente. Feuer (der Sonne), Wasser (der Wolken), Luft (des Himmels) und Erde stehen zwar eher assoziativ nebeneinander, sind aber doch im Text dem Regenbogen zugeordnet: $d a$ gestet der boge von vier furstlichen elementis, $(5,17)$ (Der Regenbogen entsteht also aus den vier fürstlichen Elementen).

Die vier Elemente werden dann auf das `Uns des Textes projiziert: Die Elemente sind es, dei uns temperent die atemzuht $(5,18)$, die uns also die Atemzüge ermöglichen (oder erleichtern). Diese Überleitung bereitet eine weitere numerische Steigerung vor: Die fünf Sinne begreifen den Regenbogen - und zwar zuerst die fünf Sinne des Leibes $(5,21)$, die aber vermögen nicht die tieffe allere dinge gruntes $(5,20)$ (die Tiefe des Grundes aller Dinge) zu begreifen, erst die fünf Sinne der Seele vermögen das Wunder Gottes zu umfassen. Der Text entwickelt also eine aufsteigende Reihe: Ein Regenbogen besteht aus zwei Elementen, zu dem ein drittes kommt und ein viertes die Zahl der Elemente komplettiert. Diese vier führen dann zur Fünf der Sinne, die alle in dem einen Wunder zusammenfallen.

Kombiniert und neuorganisiert wird hier allgemeines Wissen der Quellen im Lucidarius, der mittelhochdeutschen Übersetzung des Honorius, entstehen die Farben des Regenbogens durch die vier Elemente ${ }^{18}$ - und dies wird koordiniert

18 Vgl. Der deutsche >Lucidarius`. Hg. von Dagmar Gottschall und Georg Steer. Tübingen 1994, S. 58, 9-15. 
mit der eigenen Welterfahrung, die dezent, aber doch explizit eingebaut wird: $i c h$ waene $(5,16)$.

Auf dieser Grundlage wird ein zweiter exegetischer Ansatz eröffnet: Wasser und Feuer seien Mittel des Gerichts: Das Wasser der Sintflut, das Feuer des Weltendes $(6,7)$. Der Regenbogen wird dadurch temporalisiert. Er verweist zurück auf die Sintflut und voraus auf das Jüngste Gericht. Das >Ich positioniert sich dazwischen, - daz ergangene wizze wir, des chúmftígen g(e)warte wir $(6,17)$ (das Vergangene kennen wir, das Künftige erwarten wir) - positioniert sich im zeitlichen Gefüge der Heilsgeschichte, gleichsam nach augustinischem Zeitverständnis zwischen >Erinnern « und >Warten ` - das aber kann ich hier nur andeuten.

Was passiert hier? Das Weltwissen um den Regenbogen, die vorsichtige Spekulation des >Ich ‘ wird im Text mit dem Wissen des magezoge und der buochmeister enggeführt. So entsteht ein Text, der in der Logik der aufsteigenden Zahl eine Form sucht und findet und so die Stimme des >Ich` mit den pergamentenen Instanzen monastischer Glaubenspraxis vereint.

Meine Versuchsanordnung, von Evidenzen auszugehen, ließe sich noch weiter fortsetzen und das über eine materielle und inhaltliche Ebene hinaus. Ich will hier nur auf zwei weitere Evidenzen hinweisen, denen nachzugehen sich lohnen könnte. Es ist die Evidenz der Form und die Evidenz der Kommunikationssituation.

\section{Die Evidenz der Form}

Daz himelrîche ist in einem ganz einmaligen Metrum geschrieben. Es sind unregelmäßige Langverse, deren Anverse in der Regel einen Überschuss an Hebungen gegenüber den Abversen haben. Die Binnenzäsur wird oft pointiert durch Binnenreim. Die kürzeren Abverse lassen an die Langverse des Kürenbergers oder an die Nibelungenstrophen denken, die raumzeitlich in direkter Nachbarschaft mit dem himelrîche verwendet wurden.

Dieter Kartschoke verwies aber auch auf Parallelen zur lateinischen Metrik und konkret auf Formen der Hymnik. ${ }^{19}$ Man kann die Genese des Metrums nicht eindeutig klären, fest steht nur seine Besonderheit. Der Text gibt der Arbeit am Wortschatz, der Thematisierung des eigenen Weltwissens und der daraus entstehenden exegetischen Textpraxis eine spezifische Form, die lateinische

19 Vgl. Dieter Kartschoke: Die Metrik des Gedichts >Vom Himmelreich` im Urteil der Forschung. In: Karl-Friedrich Kraft, Eva-Maria Lill, Ute Schwab (Hg.): triuwe. Studien zur Sprachund Literaturwissenschaft. Gedächtnisbuch für Elfriede Stutz. Heidelberg 1992, S. 159-174. 
Metrik und volkssprachige Verse in einen Horizont stellt. Die repetitive Syntax, die die Synonymhäufung erzeugt, bekommt ihren eigenen Ton.

\section{Die Evidenz der Kommunikationssituation}

Gleiches gilt für die Kommunikationssituation: In den Literaturgeschichten wird das himelrîche als "gebetshaft« bezeichnet: Ein >Ich» wende sich direkt an Gott: Michil bist du herro got. Aber das stimmt nicht durchgehend. Es spricht auch ein >Wir $<$ und ein >Uns $<$ und Gott wird auch in der dritten Person thematisiert. An einer zentralen Passage beobachtet das sprechende >Ich « seine Seele und sagt: daz si mit ire gote so unde sus lantrehten muozze. (3,7f.) (Dass sie mit ihrem Gott so oder anders verhandeln muss). Die Verbalableitung von lantreht, lantrehten, begegnet uns hier zum ersten Mal. ${ }^{20}$ Die Seele muss in der Konsequenz dessen, was das >Ich sagt, mit Gott gleichsam prozessieren. Der Text ist also kein Gebet, auch wenn er so beginnt. Das >Ich verän, sondern relativiert seine Aussagen stets und das in Relation zu anderen Instanzen, wie dem Lehrer und den Gelehrten. Es entsteht ein lantrehten - ein prozesshaftes Verhandeln auch mit Gott, in der das >Ich`, wie gesagt, etwas riskiert. Es riskiert: daz ich verliese die arbeite minere anedæhte $(5,9)$.

Die Textpraxis, die wir hier greifen, ist keine literarische, sondern eine spirituelle Praxis. anedæhte, wie der Text sagt und das in Form eines Prozesses, in der sich das >Ich im Konzert bekannter Instanzen positioniert und dafür spezifische Formen findet. Diese kennen wir durchaus auch aus der Literatur. Weltbewältigung in Textform ist eben beides. Dies aber mehr als Exkurs und als Überleitung zu meiner letzten Evidenz.

\section{Die Evidenz der Baustelle}

Evidenz der Baustelle, das klingt vielleicht etwas schräg und ich muss zugeben, dass ich damit nun endgültig zur Kettensäge greife. Aber ich bleibe doch erst einmal am Text: Architektonische Details spielen im himelrîche eine besondere Rolle. Auch hier tauchen viele Begriffe zum ersten Mal auf: Beschrieben wird etwa der »Estrich« im »Himmlischen Jerusalem « (4,9): des sales estrich ist mit vehen steinen gestrowet (Der Estrich des Saales ist bestreut mit bunten, vehen,

20 Ausführlich dazu Stridde: Verbalpräsenz (s. Anm. 7), S. 80 f. 
Steinen). Die Seligen lehnen an lineberge $(8,8)$ - im Althochdeutschen übersetzt das Wort meist cancellos - »Zinnen« sind das also oder auch eine Form von »Erker« - also ein Architekturdetail, das die Vorstellung von >Lehnen gen (Komfort und Sicherheit) vereint. ${ }^{21}$ Überhaupt sind Innenräume gut: Warm, sicher, sauber. Das ist natürlich eine allgemeine Alltagserfahrung, aber im Falle der Windberger Verbindung von Exegese der Alltagserfahrung eben vielleicht auch mehr.

Die Windberger Baukunst geht konkret auf Otto von Bamberg zurück neben Biburg und Prüfening war Windberg sein drittes Projekt, dessen Fertigstellung er aber nicht erlebte: Er starb 1139. ${ }^{22}$ Die Altarweihe, die der Olmützer Bischof Heinrich Zdik am 21. und 22. Mai 1142 vollzog, hätte ihm auch kaum gefallen. Sie fand nämlich unter einem Notdach statt, denn außer dem Presbyterium und den Apsiden war der Bau noch nicht fertiggestellt. Es sollte bis 1167 dauern, bis die Ostteile einschließlich der Gewölbe vollendet waren. Am 28. November 1167 erfolgte durch Bischof Johannes von Olmütz eine zweite Weihe, die als eigentliche Kirchweihe gilt. In dieser Bauphase entstand eine Kirche für den Nonnenkonvent, die am 21. April 1158 geweiht wurde. ${ }^{23}$ Die große Klosterkirche selbst harrte aber weiter ihrer Vollendung. Deutlich ist eine Baunaht zwischen den Mauern des Lang- und Querhauses. Die neuere Forschung zeigt zusätzlich, dass der Anschluss des Langhauses durch versetzte »Wartesteine ${ }^{24}$ vorbereitet wurde. Erst um 1230 war das Langhaus dann fertiggestellt.

Wie auch immer diese Verzögerungen zustande kamen, Kloster Windberg war von seiner Gründung an nicht nur der Ort spiritueller Kontemplation, sondern auch eine Baustelle: Schmutz, Lärm, Messfeiern unter Notdächern, ohne vollendeten Kirchenraum. Im Text des himelrîche dagegen erträumt man sich eine Sicherheit und einen Komfort von Innenräumen: Greift das die Alltagserfahrung der Baustelle auf? Stattet das die Zumutungen des täglichen Baubetriebs

21 Dazu ausführlich Stephan Müller: Minnesang im Himmelreich? Über Örtlichkeiten literarischer Kommunikation an den Grenzen des Höfischen beim Kürenberger, in der `Kudrun`, im >Dukus Horant` und im >himelrîche`. In: Beate Kellner, Ludger Lieb, Peter Strohschneider (Hg.): Literarische Kommunikation - soziale Interaktion. Frankfurt a.M. u. a. 2001, S. 51-71.

22 Vgl. Alexandra Fink: Romanische Klosterkirchen des hl. Bischofs Otto von Bamberg (11021139): Studien zu Bauherr und Architektur. Petersberg 2001, S. 175.

23 Vgl. Norbert Backmund: Windberger Kirchen und Kapellen. In: Verhandlungen des Historischen Vereins für Niederbayern 98 (1972), S. 5-20, hier S. 11. Diese Kapelle wurde 1849 wieder abgebrochen.

24 Fink: Romanische Klosterkirchen (s. Anm. 22), S. 175. Abgebildet sind Baunaht und Wartesteine bei Peter Schwarzmann: Die Hirsauer Reform und die Kirchenbauten Bischof Ottos I. für die Klöster Windberg, Biburg und Prüfening. In: 850 Jahre Prämonstratenserabtei Windberg. München, Zürich 1993, S. 40-62, hier Abb. 43-45. 
nicht mit einer spirituellen Dimension der Hoffnung aus? Geht mit ihm nicht auch ein Stück weit das in Erfüllung, was uns das >Himmlische Jerusalem`verspricht?

Fassen wir die Sache noch etwas weiter: Die 50 Kilometer entfernte Bischofsstadt Regensburg müssen wir uns zur Zeit der Entstehung des himelrîche so vorstellen, wie weiland das Konzert der Kräne am Potsdamer Platz. Nach der extremen Trockenheit von 1135 war bis 1146 die Steinerne Brücke entstanden, ein architektonisches Wunderwerk, das die Stadt bis in die Neuzeit zu einem Verkehrsknoten machte: Schon 1149 versammelte man sich dort zum zweiten Kreuzzug, Napoleons Armeen marschierten tagelang über die Brücke, die Nazis versuchten sie zu sprengen. Am 22. und 23. April 1945 wurden auf Befehl des Gauleiters Ludwig Ruckdeschel zwei Pfeiler der Brücke gesprengt, um den Vormarsch der Amerikaner zu verzögern. In Regensburg formierte sich eine Versammlung, die den Unmut darüber zum Ausdruck brachte. Domprediger Johann Maier versuchte zu schlichten. Der anwesende Gauleiter Ruckdeschel forderte seine sofortige Erhängung im Angesicht der Menge, aber immerhin kam es zum Standgericht. Johann Maier wurde noch am gleichen Abend verurteilt und am nächsten Morgen gehenkt. Am Abend dieses Tages zogen die Amerikaner über die Brücke. Der Regensburger Bischof Michael Buchberger hielt sich bei der Verurteilung Maiers verdächtig still und zog sich dezent in einen Keller zurück, in dem er sich versteckte. Gauleiter Ludwig Ruckdeschel starb 1986 - wegen der etwas übermotivierten Hinrichtung wurde er zu 8 Jahren Haft verurteilt. Nach seiner sehr vorzeitigen Entlassung war er als Führer für prominente Gäste bei Volkswagen in Wolfsburg tätig. 1959 erhielt Bischof Michael Buchberger den Bayerischen Verdienstorden und starb 1961 in Straubing (wenige Kilometer von Windberg, wo unser himelrîche entstand). 2005 wurde Johann Maier die Ehre eines Grabes in der Domkrypta zuteil - dort liegt er jetzt neben den Regensburger Bischöfen.

Aber nach diesem kurzen Exzess von Assoziationen, die die Baugeschichte der Steinernen Brücke bis in die Moderne perspektivierte und andeuten sollte, welche kulturhistorische Dimension der Blick auf ein Bauwerk freisetzen und auf welche Wege und vielleicht auch Abwege das eigentliche Thema führen kann, zurück ins Mittelalter und zum himelrîche. Der Bau der Steinernen Brücke war ein komplexes Großprojekt. Das Regensburger Donauufer musste völlig umgebaut werden. Die römische via praetoria wurde überbaut, sodass die porta praetoria verschwand. Und mehr noch: 1156 und 1172 brannte der romanische Dom in Regensburg ab und wurde jeweils wiederaufgebaut. 1166 brannte das Langhaus von St. Emmeram und man begann mit einer Neugestaltung des Kirchenraums. Und: In Regensburg steht mit Prüfening der Vorbildbau für Windberg. 
Architektur wird im Mittelalter in der Regel als Ganzes gedeutet: »Die Kathedrale als Zeitenraum «. ${ }^{25}$ Tatsächlich aber begegneten die Kathedralen den Menschen als Baustellen, die sie meist bis in die Moderne blieben. Bauen ist Gottesdienst und prägende Erfahrung des Alltags zugleich. Baut das himelrîche auch diese Alltagserfahrung in die Formen traditioneller Exegese ein? Diese Frage ergibt sich für mich aus der Beschreibung einer Textpraxis, die Alltagserfahrung auf eine spezifische Weise integriert - nur gebunden an diese Beobachtung ergab sich für mich eine Evidenz. Das sagt zunächst nichts über das Verhältnis von Architektur und Literatur, aber bringt eine neue Perspektive für die Beschreibung der Textpraxis des himelrîche bei.

\section{Schluss}

Zum Schluss nun nochmal ein Blick über meine Evidenzen und zur Frage nach den Möglichkeiten, sie miteinander in Bezug zu setzen. Hervorheben möchte ich dabei eine Differenz, die ich versuchsweise einmal (mit Anleihe aus der Sprache des Rechts), als die Differenz zwischen >Indizien` und >Beweisen ` beschreiben will. Ein >Beweis` ist das positive Ergebnis einer Feststellung von Tatsachen. Das positive Ende eines Beweisverfahrens, wobei entscheidend ist, dass die >Beweise` zulässig sind und dass der Sachverhalt beweisbedürftig ist. Ein >Indiz^ dagegen zeigt an, dass ein Sachverhalt mit einer hohen Wahrscheinlichkeit zutrifft. Es handelt sich um Aussagen, die nur mittelbar auf einen Sachverhalt verweisen. >Indizien` sind Anzeichen für >Beweise`, aber keine eigentlichen >Beweise`. Das `Indiz` ist also mehr als nur eine reine Behauptung, aber weniger als ein positiver >Beweis<.

Lege ich diese Differenz auf meine Evidenzen um, dann scheint es mir, als ob auch hier >Beweise « von >Indizien` zu unterscheiden sein könnten. Die Evidenz etwa des Materials ergab sich nur aus dem `Beweis` der Schreiberidentität, der Austausch mit Oberaltaich aus dem Lesartenvergleich der Moralia-Handschriften, die Untersuchung zu den Quellen operiert mit inhaltlichen Identitäten. Ähnlichkeiten würden hier keine belastbare Aussage ergeben und kein Argument begründen. Solche >Beweise können nur solche sein, wenn man sie an etwas Normativem messen kann: An Vorstellungen von richtig und falsch, von möglich und unmöglich. Diese Normen sind verhandelbar und variieren im Zuge der Forschung (sie sind sozusagen die Grundlagen für die Beweisanträge),

25 So der Titel des zum Klassiker gewordenen Aufsatzes von Friedrich Ohly: Die Kathedrale als Zeitenraum. Zum Dom von Siena. In: Frühmittelalterliche Studien 6 (1972), S. 94-158. 
aber man braucht sie, sonst werden philologische Argumente zur Plauderei. Man muss also immer auch überprüfen, was die Basis philologischer Argumente war: Die Paläographie hat sich weiterentwickelt, die historische Sprachforschung hat ihre Aussagen modifiziert (und tut das weiterhin), etc.

Natürlich werden diese >Beweise nur als Teil von hermeneutischen Prozessen aussagekräftig. Eine Schreiberidentität besagt so wenig wie identische Lesarten und Quellennachweise. Erst im Zuge der Interpretation wird daraus ein Bild der Text- und Schreibpraxis in Windberg, wird daraus eine Deutung des himelrîche.

Es gab aber auch >Indizien ‘ etwa für die Evidenz des Alltags oder der Baustelle. Die Nennung von architektonischen Details in einem Text besagt erst einmal gar nichts. Erst die Untersuchung, wie der Text Alltag thematisiert und welches Konzept von theologischer Text- und Lebenspraxis er damit verfolgt, macht aus dem Hinweis auf die Nennung von architektonischen Details ein mögliches Argument. Dabei konnte ich mich nicht auf eine gegebene Relevanz zurückziehen (wie für die Metrik, die Überlieferung, etc.), sondern musste im Zuge der Interpretation eine Relevanz der architektonischen Details, eine Relevanz des Bauens und der Baustelle auch mit Blick auf die Realhistorie erst begründen.

Überblicke ich den Alltag unserer Forschung, würde ich sagen, dass die, die sich als Philologen bezeichnen, stärker mit >Beweisen` operieren. Die philologischen >Beweise < können an und für sich und getrennt voneinander erhoben werden. Paläographie, Textkritik, metrische Untersuchungen, das kann man für sich genommen betreiben und die Ergebnisse dann in hermeneutische Prozesse einbauen. Das heißt nicht, dass die >Beweise der Philologie nicht hinterfragbar und unveränderbar sind oder, vom empirischen Wert her gesehen, eine engere Verbindung zur Welt, oder sagen wir ruhig euphorisch Wahrheit aufweisen. Vielmehr sind es die Konventionen und die Formen der Akzeptanz der Philologie, die sie zu für sich selbst stehenden >Beweisen`machen. Diese Konventionen und Fragen der Akzeptanz können sich aber natürlich ändern, so wie aus den ehernen Beweisen der Konjekturalkritik teilweise unlautere, aus ahistorischen Idealvorstellungen (etwas von Stil und Metrik) hergeleitete Setzungen wurden - um nur ein Beispiel zu nennen. >Indizien` dagegen können nicht für sich stehen, sie werden nicht um ihrer selbst willen erhoben, sondern sie ergeben erst im Zuge hermeneutischer Prozesse Sinn. Mir scheint, als ob eine kulturwissenschaftliche Literaturwissenschaft stärker mit solchen `Indizien ‘ arbeitet. Die Beobachtung der Nennung von architektonischen Details wird erst im Zuge der Interpretation zum Argument. Es würde Sinn machen, Wortschatz der Architektur zu sammeln, wenn man eine Wortschatz-Untersuchung machen will, aber texthermeneutisch wäre das blind, solange man nicht sagen kann, warum 
die architektonischen Details für das Verständnis des Textes wichtig sind. Jede Rechnung, jedes Abgabenverzeichnis könnte die gleichen Gegenstandsbereiche wie das himelrîche adressieren, ohne dass man sinnvoll nach einer Hermeneutik der Baustelle fragen könnte.

Es geht bei meiner Differenzierung von >Beweisen` und >Indizien ‘ übrigens nicht darum, das eine vor dem anderen $\mathrm{zu}$ würdigen, etwa >Beweise $<$ vor $>$ Indizien . Beides führt am Ende zu einer Verurteilung und Indizienprozesse sind viel faszinierender als Beweisverurteilungen (die dafür aber weniger anfällig für Fehlentscheidungen sind und die Richter besser schlafen lassen). Wenn ich die >Indizien ‘ stärker auf der Seite einer kulturwissenschaftlichen Philologie sehe, dann bedeutet das, dass diese ihre Gegenstände als solche erst plausibel und zum Gegenstand einer wie auch immer gearteten Hermeneutik machen muss. Die >Beweise` der Philologie können für sich stehen. Sie können immer belastet werden. Sie entspringen hergebrachten Formen der Text- und Spracharbeit und sind in ihrer Relevanz eingeführt, was natürlich nicht heißt, dass diese Relevanz nicht modifizierbar ist. Man denke nur an alte >Beweise der Konjekturalkritik, die auf einer Basis solche waren, die inzwischen gründlich demontiert ist. Ein kulturwissenschaftlicher Indizienbeweis ist indes voraussetzungsreicher. Man muss begründen, warum etwas für das Verständnis eines Textes relevant ist, welche Rolle Motive des Alltags im himelrîche für das Verständnis des Textes spielen.

Ähnliches hat Hartmut Böhme für die Kulturwissenschaft ganz allgemein beschrieben - und zwar am Beispiel der »Baustelle $« .{ }^{26}$ Ein Student hatte in einem Seminar »Stadt-Räume« ein Referat zum Thema »Die Baustelle« geplant und bemerkt, dass es die >Baustelle` als Gegenstand der Kulturwissenschaft noch nicht gibt. Jeder weiß, was eine Baustelle ist, in vielen Disziplinen wird mit Baustellen operiert und werden Baustellen thematisiert, aber um die Baustelle zum Gegenstand der Kulturwissenschaft zu machen, muss der bekannte Gegenstand neu perspektiviert werden. Böhme nennt »rhapsodisch« eine Reihe von Punkten, die dabei eine Rolle spielen könnten. Baustellen sind ephemere Institutionen, die sich von einer alltäglichen Kultur abheben. Sie bilden spezifische (oft multikulturelle) Vergemeinschaftungen aus und eigene Symbolsysteme (wie Feste). Baustellen werden von außen als Besonderheit wahrgenommen und das mit einem durchaus ästhetischen Potential (Stichwort Schaustelle). Baustellen haben eine eigene sich wandelnde Faszinationsgeschichte und potentiell drastische soziale und kulturelle Auswirkungen, wenn in ihnen etwa technische Innovatio-

26 Hartmut Böhme: Zur Gegenstandsfrage der Germanistik und Kulturwissenschaft. In: Jahrbuch der deutschen Schillergesellschaft XLII (1998), S. 476-485. Ich fasse die Argumentation Böhmes im folgenden Abschnitt zusammen. 
nen auf die sozialen Hintergrundstrukturen, die die Baustelle herausbilden, einwirken.

In diesem Verständnis ist die Kulturwissenschaft eine Wissenschaft der Welterklärung, die natürlich ihren Reiz und ihre Berechtigung hat. Als germanistischer Mediävist würde ich mich gerne daran beteiligen, aber muss eben einschränken, dass (1) schon die genannten Aspekte der Baustelle historisch extrem variabel sind und dass (2) ich natürlich nur über Texte sprechen kann, die historischen Objekte entziehen sich dagegen meinem Zugriff. Böhme sieht das anders und betont, dass sich die Kulturwissenschaft nicht auf textuelle Objekte beschränken darf. Das ist für sein Verständnis von Kulturwissenschaft richtig und ebenso ist richtig, dass sich auch die Literaturwissenschaft nicht auf die Beschreibung von Texten beschränken muss (sie kann aber, wenn sie will). Der Unterschied ist der Ausgangspunkt. Ich meine stets vom Text ausgehen zu müssen. Die darin behandelten Objektbereiche sind natürlich auch Gegenstand anderer Disziplinen, mit denen man zusammenarbeiten kann. Für den Objektbereich >Bausteller gibt es Bauhistoriker, Archäologen, Kunsthistoriker, Philosophen, Theologen, die sich aber sinnvoll nur unterhalten können, wenn sie eine gemeinsame Fragstellung verbindet. Kulturgeschichtlich weiter gefasst kann die Evidenz der Baustelle nur im Gespräch mit anderen Disziplinen Sinn abwerfen: Die Architekturgeschichte muss die Realien bewerten, die Kunstgeschichte kann sagen, ob und wie die Baustelle als ikonographisches Motiv verwendet wird, die Religions- oder Philosophiegeschichte kann fragen, ob die Baustelle als Denk- oder Argumentationsmuster wirksam wird, usw. Und dann muss man sich fragen, was man daraus macht: Arbeitet man an einer Diskursgeschichte des Phänomens? Dann hilft Foucault. Geht es darum, wie unbelebte Dinge mit geistigen und sozialen Prozessen vernetzt sind, dann wird man bei Bruno Latour eine methodische Klammer suchen. Ohne solche Klammern jedoch führt die Evidenz ins Nichts.

Eine kulturgeschichtliche Untersuchung kann durchaus mit Ähnlichkeiten operieren, mit `Indizien ‘ also, wie die Beobachtungen zur Baustelle: Man muss nur deutlich machen, welchen Status diese >Indizien` haben und in welchen Kontexten sie $\mathrm{zu}$ >Beweisen 'werden: Ist die Baustelle ein Motiv? Steht sie für einen Diskurs? Bringt sie Objekte in einem Netzwerk hervor? All das kann man legitimer Weise fragen. Aber man muss eben damit auch die Reichweite der Antworten bedenken. Die Philologie ist da (auch wenn ihr Ruf ein anderer ist) offener, denn natürlich kann ein Kulturwissenschaftler mit philologischen >Beweisen operieren - aber die Frage, wie und wann ein Philologe mit kulturwissenschaftlichen >Indizien` argumentieren kann, ist restriktiver zu beantworten.

Vor allem aber ist immer mit zu bedenken, wie man die Ebene der historischen Objekte und die Ebene der Texte und Bilder aufeinander bezieht. Das ist 
natürlich das Grundproblem der Literaturwissenschaft schlechthin - die gute alte Vermittlungsproblematik. Man kann sie nicht lösen, aber man kann versuchen, auf das Problem zu reagieren, etwa indem man klarstellt, dass historische Objekte mit Vorstellungen von denselben verbunden sind und Texte Vorstellungen historischer Objekte generieren. Dann arbeitet man an einer Geschichte des Imaginären, in der Texte und Objekte friedlich nebeneinander existieren können - solange man nicht in die Vorstellung abgleitet, das eine sei mit dem anderen identisch, so sehr die Literatur uns auch dazu einlädt. Das sind natürlich Trivialitäten auf Einführungsniveau, die man voraussetzen sollte (ohne laufend Roland Barthes und Co. in die Fußnoten setzen zu müssen weshalb ich hier darauf verzichte), aber mir scheint, dass im Kontext einer kulturwissenschaftlichen Herangehensweise an die Texte oft vergessen wird, dass es sich um Texte handelt. Wenn man Assoziationen, Implizites, Eindrücke radikal zu Argumenten macht, ist es schon geschehen. Aber das gilt auch für die Erhebung philologischer >Beweise`, wenn man sich nicht klarmacht, dass sie nicht für sich selbst sprechen, sondern nur im Kontext einer wie auch immer gearteten Methodik Sinn abwerfen.

Damit abschließend doch noch zum Thema: Was kommt nach der Kulturgeschichte? Es kommt natürlich die Kulturgeschichte! Wenn ich es richtig sehe, war das schon immer so, so wie nach der Sozialgeschichte (um die Frage in der Terminologie von Martin Huber und Gerhard Lauer zu stellen) eine neue Form der Sozialgeschichte $\mathrm{kam}^{27}$ und wiederkommen wird - unterbrochen von Ruhepausen aller möglicher Formen der Textimmanenz. Oder: So wie psychologische Ansätze, die mal erlaubt, mal verboten sind, mal mit Freud dann mit Lacan - und natürlich hatten die Menschen des Mittelalters eine Psyche und natürlich kann man versuchen, diese Ebene fruchtbar zu machen, aber die Versuche sind eben jeweils voraussetzungsreich und erfordern eine (oft vielleicht dann doch nicht mögliche) Historisierung und eine methodische Positionierung, wie man sich das Verhältnis zwischen Text und Welt (und Theorie) vorstellt. Die Fragen der Kulturgeschichte sind wie Schlaghosen - sie kommen immer wieder (und selig der, der immer eine Schlaghose im Schrank hat - nicht um sie zu tragen, sondern um sich daran zu erinnern, dass man sie einst trug). Es kommen immer neue kulturgeschichtliche Fragestellungen und Instrumente, die für sich genommen immer ihre Berechtigung haben, deren Ergebnisse nur nicht unhinterfragt in allen Kontexten als Ar-

27 Als Beispiel dafür sehe ich die Arbeiten von Jan-Dirk Müller, die an den möglichen Verbindungen von Kulturmustern und Textmustern laborieren, also auf einer neuen methodischen Ebene an der Vermittlungsproblematik als altem Kernproblem der Sozialgeschichte weiterarbeiten und monographisch in seine »höfischen Kompromisse« gemündet sind. Jan-Dirk Müller: Höfische Kompromisse. Acht Kapitel zur höfischen Epik. Tübingen 2007. 
gumente genommen werden dürfen. Es kommt darauf an, Kulturgeschichte nicht um ihrer selbst willen zu betreiben; zumindest Philologen sollten das nicht tun, Kulturwissenschaftlerinnen und Kulturwissenschaftler müssen das tun. Die philologische Objektebene muss der Text bleiben, solange das so ist, kann man mit ihm machen, was man will. Solange man vom Text ausgeht und offenlegt, was man mit ihm tut, ist alles erlaubt. Nicht mehr, aber auch nicht weniger versuchte ich anhand meines Beispiels vorzuführen. Die Kulturwissenschaft hat dabei viele neue Perspektiven eingebracht und wird weitere einbringen, aber Textwissenschaftler dürfen dabei nicht die Kultur selbst auf der Objektebene behandeln, auch wenn die Literatur alles daransetzt, uns dazu zu verleiten. 